\section{Gastos com a assistência ao parto: comparação entre as coortes de nascimento de Pelotas dos anos de 2004 e 2015, Rio Grande do Sul, Brasil}

\author{
Expenditures on childbirth care: a comparison \\ of the 2004 and 2015 birth cohorts in Pelotas, \\ Rio Grande do Sul State, Brazil
}

\section{Gastos con la asistencia al parto: comparación entre las cohortes de nacimientos de Pelotas (Rio Grande do Sul, Brasil) desde el año 2004 y 2015}

\author{
Marília Cruz Guttier 1 \\ Cesar Augusto Oviedo Tejada 2 \\ Fernando C. Wehrmeister 1 \\ Mariângela Freitas Silveira 1 \\ Marlos R. Domingues 3 \\ Aluísio J. D. Barros 1 \\ Iná S. Santos 1 \\ Alícia Matijasevich 4 \\ Diego G. Bassani 5 \\ Andréa Dâmaso Bertoldi 1
}

doi: $10.1590 / 0102-311 \times 00120019$

\section{Resumo}

Apesar de a maioria dos partos no Brasil ser financiada pelo Sistema Único de Saúde (SUS), existem gastos diretos (pessoais privados) envolvidos no nascimento. Este estudo visa a comparar o desembolso materno para financiar os partos das crianças pertencentes às coortes de nascimento de Pelotas de 2004 e 2015. Foram utilizadas informações coletadas logo após o nascimento e aos três meses de idade. As variáveis analisadas incluem informações sociodemográficas, econômicas, cobertura por plano privado de saúde e despesas relacionadas ao parto. Os valores de 2004 foram ajustados pelo Índice Nacional de Preços ao Consumidor Amplo (IPCA). Observou-se aumento na posse de planos de saúde de 33,4\% (IC95\%: 31,9-34,9) para 45,1\% (IC95\%: 43,6-46,7) no período analisado e este esteve diretamente associado à posição econômica das famílias ( $p<0,001)$. Ocorreu um aumento na média dos gastos com hospitalização para o parto de $R \$ 60,38(D P=288,66)$ para $R \$ 171,15(D P=$ 957,07), e nos gastos adicionais com médicos de $R \$ 191,60(D P=612,86)$ para $R \$ 1.424,80(D P=4.459,16)$ entre as mães que se internaram pelo plano privado de saúde (e não houve diferença significativa nestes gastos entre as mães que optaram pelo parto particular). Houve aumento importante no gasto com a assistência ao parto principalmente entre as mães que se internaram pelo plano privado de saúde.

Equidade em Saúde; Parto Obstétrico; Serviços de Saúde Materna; Gastos em Saúde; Estudos Longitudinais

\section{Correspondência}

M. C. Guttier

Programa de Pós-graduação em Epidemiologia, Faculdade de Medicina, Universidade Federal de Pelotas.

Rua Marechal Deodoro 1160, 3o piso, Pelotas, RS 96020-220, Brasil.

maguttier@gmail.com

1 Programa de Pós-graduação em Epidemiologia, Universidade Federal de Pelotas, Pelotas, Brasil.

2 Programa de Pós-graduação em Organizações e Mercados, Universidade Federal de Pelotas, Pelotas, Brasil.

3 Programa de Pós-graduação em Educação Física, Universidade Federal de Pelotas, Pelotas, Brasil.

4 Faculdade de Medicina, Universidade de São Paulo, São Paulo, Brasil.

${ }^{5}$ Hospital for Sick Children, University of Toronto, Toronto, Canada. 


\section{Introdução}

Os gastos, público e privado, com saúde têm aumentado consideravelmente em vários países, inclusive no Brasil 1. A Pesquisa de Orçamentos Familiares (POF), em suas últimas edições, tem apresentado um aumento nos gastos dos brasileiros com assistência à saúde. Na última edição dessa pesquisa (POF 2017-2018), gastos com assistência à saúde tiveram participação na despesa média mensal familiar de $8 \%$. Cerca de $2 \%$ das despesas médias mensais das famílias ocorrem com planos privados de saúde 2,3.

Os dados da Pesquisa Nacional por Amostra de Domicílios (PNAD), realizada nos anos de 1998 e 2003, e da Pesquisa Nacional de Saúde (PNS), conduzida em 2013, demonstraram que aproximadamente um quarto da população brasileira usou o setor de saúde suplementar (planos de saúde ou saúde privada) pelo menos seis meses anteriores à entrevista 4,5,6. De acordo com a Agência Nacional de Saúde Suplementar (ANS), a taxa de cobertura dos planos de assistência médica em dezembro de 2015 era de $25,8 \%$, sendo as regiões Sudeste e Sul as que apresentaram as maiores coberturas 7 .

No Brasil, algumas políticas foram implantadas com o objetivo de humanizar o parto, reduzir o número de cesárias e regulamentar a cobrança do acompanhamento do trabalho de parto $8,9,10$. Até recentemente, as gestantes que possuíam plano de saúde com cobertura obstétrica podiam escolher o obstetra de sua preferência, o qual as acompanharia em todo o procedimento pré-natal e, ao final da gestação, realizaria o parto, não tendo de pagar qualquer outro valor além da mensalidade do plano de saúde 11. Porém, essa realidade vem sendo alterada com a cobrança pelos médicos de uma taxa extra, a ser paga pela gestante diretamente para o obstetra, denominada taxa de disponibilidade ou taxa de acompanhamento para parto, como condição para que o especialista que a acompanhou no pré-natal fique "disponível" para a realização do parto 11,12.

Nesse sentido, este estudo visa a comparar os gastos relacionados à assistência com parto entre as coortes de nascimento de Pelotas, Rio Grande do Sul, de 2004 e 2015, verificando se ocorreu alguma mudança no padrão do desembolso familiar entre as mulheres cuja fonte de financiamento para o parto era plano privado de saúde ou com financiamento próprio.

\section{Método}

Nos anos de 2004 e 2015 todos os nascidos vivos de mães residentes na área urbana da cidade de Pelotas eram elegíveis para participar do estudo de coorte de nascimento dos respectivos anos. Em 2004, 99,31\% das mães consentiram que seus filhos fossem arrolados pelo estudo. Em 2015, 4.275 (98,75\%) deram o consentimento para a participação dos filhos na pesquisa. As crianças da coorte de 2004 foram avaliadas ao nascer e acompanhadas aos 3, 12, 24 e 48 meses, e aos 6 e 11 anos de idade; e as da coorte de 2015 foram avaliadas ao nascer e acompanhadas aos 3, 6, 12 e 24 meses. Foram empregados os mesmos métodos para a coleta de dados nos dois estudos. Detalhes sobre a metodologia usada em 2004 e 2015 estão disponíveis em outros artigos 13,14,15 e os questionários utilizados estão disponíveis no link: http://www.epidemio-ufpel.org.br/site/content/estudos/index.php. Para este estudo foram usados os dados coletados ao nascer e aos três meses das duas coortes.

\section{Variáveis coletadas ao nascer}

Em ambos os estudos, durante a sua permanência no hospital, as mães foram convidadas a participar do trabalho e, depois de assinarem o termo de consentimento informado, foram entrevistadas por entrevistadoras treinadas. Foi utilizado um questionário estruturado contendo informações de identificação, parto e saúde do recém-nascido, bem como características socioeconômicas da família e demográficas maternas (cor da pele e idade). A cor da pele materna foi obtida por autorrelato utilizando-se as seguintes categorias: branca, preta, parda, amarela e indígena. Devido à baixa frequência de mães que se autodeclararam pardas, amarelas e indígenas, estas foram posteriormente classificadas na categoria "mista".

A renda familiar foi calculada por meio da soma dos valores reportados pelas mães quanto ao rendimento de cada pessoa que vivia na residência no mês anterior à entrevista. Os valores 
foram transformados para salários mínimos utilizando-se os valores vigentes nos respectivos anos: R\$ 260,00 em 2004 e R\$ 788,00 em 2015.

Também foi usado o Indicador Econômico Nacional (IEN), criado por meio da análise de componentes principais, utilizando-se 12 itens (televisão, rádio, carro...) e a escolaridade do chefe da família. Esse indicador foi calculado para a coorte de 2004 e 2015 e dividido em quintis levando-se em consideração os pontos de corte da distribuição do indicador para a população de Pelotas nos respectivos anos. Então, mães no primeiro quintil pertencem a $20 \%$ da população mais pobre da cidade e não da amostra. $\mathrm{O}$ mesmo acontece para os demais quintis 16 .

\section{Variáveis coletadas aos 3 meses}

Informações sobre o financiamento do parto, cobertura do plano de saúde e demais gastos foram coletadas no acompanhamento de três meses. Nesse acompanhamento, foi perguntado sobre a fonte de financiamento para o parto baseando-se na questão se a internação para o parto havia sido pelo Sistema Único de Saúde (SUS), plano de saúde ou particular. Qualquer contrato com cobertura de saúde foi considerado como plano de saúde. Além disso, as mães foram questionadas sobre a cobertura do plano de saúde, incluindo para consultas médicas, odontológicas, exames e internações.

Os desfechos estudados, em ambos os anos, foram "gastos com hospitalização" e "gastos com médicos”. Para as mães que tiveram o parto coberto por plano de saúde, esses gastos foram investigados por meio das seguintes perguntas: "A Sra. pagou alguma diferença ou adicional pelo parto ao hospital? Quanto?” e “A Sra. pagou alguma diferença ou adicional para os médicos? Quanto?”. Para mães que tiveram o parto com financiamento particular, os gastos foram avaliados pelas seguintes perguntas: “Quanto a Sra. pagou aos médicos, incluindo obstetra, anestesista, pediatra e outros?”, “Quanto a Sra. pagou ao hospital?”, “Quanto a Sra. pagou em outras despesas, como transporte de ambulância, taxas extras no hospital etc.?”

Foram realizadas análises descritivas dos desfechos e análises bivariadas utilizando-se o teste quiquadrado de heterogeneidade e de tendência linear para a comparação de proporções de acordo com as variáveis independentes, em cada ano de estudo.

Posteriormente, foram analisadas as mudanças nas proporções dos desfechos entre os anos estudados, para cada estrato das variáveis independentes. O teste qui-quadrado de Pearson foi utilizado para avaliar a significância estatística dessas mudanças. O teste $\mathrm{t}$ foi empregado para a comparação das médias de gastos entre os dois períodos. Como os valores de renda e de gastos estão fortemente influenciados pela presença de inflação, para a análise os valores de renda e gastos de 2004 e 2015 foram deflacionados pelo IPCA para valores em Reais (R\$) de dezembro de 2015, data que corresponde aos últimos dados da pesquisa (IPEADATA. Taxa de câmbio nominal 2018. http://ipeadata.gov.br/ ExibeSerie.aspx?serid=38389).

Todos os acompanhamentos da coorte de 2004 foram aprovados pelo Comitê de Ética em Pesquisa, Faculdade de Medicina, Universidade Federal de Pelotas (UFPel - 4.06.01.113). Os acompanhamentos da coorte de 2015 foram aprovados pelo Comitê de Ética em Pesquisa da Faculdade de Educação Física, UFPel (CAAE: 26746414.5.0000.5313). Em ambas as coortes, as mães ou responsáveis assinaram o Termo de Consentimento Livre Esclarecido no hospital de nascimento no início de cada acompanhamento.

\section{Resultados}

Foram acompanhadas, aos três meses das coortes de 2004 e 2015, 3.985 (95,2\%) e 4.229 (97,2\%) crianças e suas mães, respectivamente. A descrição das características maternas nos dois trabalhos está na Tabela 1. Em ambos os anos, a maioria das mães se autodeclarou de cor branca e quase 50\% tinham entre 20 e 29 anos de idade. Houve uma diminuição na proporção de mães menores de 19 anos (de 19\% em 2004 para 14,6\% em 2015) e aumento na de mães entre 30 e 39 anos (de 27,9\% para 35,1\%, respectivamente). Além disso, houve uma melhora no nível educacional na distribuição da renda na coorte de 2015, em que 31\% das mães tinham mais de 12 anos de escolaridade (aumento de 20,9p.p. - 
Tabela 1

Descrição das mães das coortes de nascimento de Pelotas, Rio Grande do Sul, Brasil, de 2004 (N = 4.189) e 2015 (N = 4.219), por ocasião do parto.

\begin{tabular}{|c|c|c|c|}
\hline Variáveis & $\begin{array}{l}2004 \\
\text { n (\%) }\end{array}$ & $\begin{array}{l}2015 \\
\text { n (\%) }\end{array}$ & Valor de p \\
\hline Cor da pele & $(n=4.141)$ & $(n=4.212)$ & $<0,001$ \\
\hline Branca & $2.556(61,7)$ & $2.982(70,8)$ & \\
\hline Preta & $682(16,5)$ & $661(15,7)$ & \\
\hline Mista & $903(21,8)$ & $569(13,5)$ & \\
\hline Idade (anos) & $(n=4.187)$ & $(n=4.218)$ & $<0,001$ \\
\hline $0-19$ & $796(19,0)$ & $618(14,6)$ & \\
\hline $20-29$ & $2.085(49,8)$ & $1.995(47,3)$ & \\
\hline 30-39 & $1.170(27,9)$ & $1.480(35,1)$ & \\
\hline 40 ou mais & $136(3,3)$ & $125(3,0)$ & \\
\hline Escolaridade materna (anos completos) & $(n=4.147)$ & $(n=4.217)$ & $<0,001$ \\
\hline $0-4$ & $647(15,6)$ & $387(9,2)$ & \\
\hline $5-8$ & $1.711(41,3)$ & $1.084(25,7)$ & \\
\hline $9-11$ & $1.372(33,1)$ & $1.441(34,2)$ & \\
\hline 12 ou mais & $417(10,1)$ & $1.305(31,0)$ & \\
\hline Renda familiar (salários mínimos) & $(n=4.177)$ & $(n=3.965)$ & $<0,001$ \\
\hline$<1$ & $1.719(41,1)$ & $246(6,2)$ & \\
\hline $1-2,9$ & $1.812(43,4)$ & $2.042(51,5)$ & \\
\hline $3-5,9$ & $442(10,6)$ & $1.113(28,1)$ & \\
\hline $6-9,9$ & $133(3,2)$ & $314(7,9)$ & \\
\hline 10 ou mais & $71(1,7)$ & $250(6,3)$ & \\
\hline IEN (quintis) & $(n=4.186)$ & $(n=4.073)$ & $<0,001$ \\
\hline Primeiro (mais pobre) & $1.006(24,0)$ & $818(20,1)$ & \\
\hline Segundo & $859(20,5)$ & $821(20,2)$ & \\
\hline Terceiro & $905(21,6)$ & $810(19,9)$ & \\
\hline Quarto & $745(17,8)$ & $815(20,0)$ & \\
\hline Quinto (mais rico) & $671(16,0)$ & $809(19,9)$ & \\
\hline Posse de plano de saúde & $(n=3.945)$ & $(n=4.052)$ & $<0,001$ \\
\hline Não & $2.626(66,6)$ & $2.223(54,9)$ & \\
\hline Sim & $1.319(33,4)$ & $1.829(45,1)$ & \\
\hline
\end{tabular}

IEN: Indicador Econômico Nacional.

Nota: valor de p refere-se ao teste qui-quadrado de Pearson.

* Indicador de riqueza baseado em índice de ativos utilizado para classificar famílias de acordo com sua distribuição na cidade de Pelotas.

pontos percentuais), diminuição de 34,9p.p. na proporção de menos de um salário mínimo e aumento de 17,5p.p. na proporção de mães recebendo entre 3 e 5,9 salários. (Tabela 1).

Entre 2004 e 2015, houve um aumento de 11,7p.p. na cobertura por plano de saúde (de 33,4\% para $45 \%$, respectivamente) (Tabela 1) e, nos dois períodos, a cobertura esteve fortemente dependente da renda familiar ( $\mathrm{p}<0,001$ para ambos os períodos, dado não apresentado em tabela).

A Tabela 2 apresenta a comparação da posse de plano privado de saúde e da cobertura dos planos para hospitalização entre as mães participantes das duas coortes. Observou-se um aumento significativo na posse de plano privado de saúde. Em 2004, 33,4\% das mães disseram ter plano privado de saúde, já em 2015 eram 45,1\%. Não houve diferença significativa na posse de plano de saúde entre as mães com 19 anos ou menos, até 11 anos de estudos e no quintil mais rico. Entre as mulheres com 12 anos ou mais de educação observou-se redução significativa na cobertura por plano privado de saúde (Tabela 2). 
Tabela 2

Comparação da posse de plano privado de saúde e cobertura do plano de saúde para hospitalização entre mães das coortes de nascimento de Pelotas, Rio Grande do Sul, Brasil, de 2004 e 2015, estratificada pelas variáveis socioeconômicas.

\begin{tabular}{|c|c|c|c|c|c|c|}
\hline \multirow[t]{2}{*}{ Variáveis } & \multicolumn{3}{|c|}{ Posse de plano de saúde } & \multicolumn{3}{|c|}{ Cobertura do plano de saúde para hospitalização } \\
\hline & 2004 & 2015 & Valor de $p$ & 2004 & 2015 & Valor de $p$ \\
\hline \multicolumn{7}{|l|}{ Cor da pele } \\
\hline Branca & $978(40,5)$ & $1.465(51,2)$ & $<0,001$ & $561(59,2)$ & $875(60,2)$ & 0,646 \\
\hline Preta & $128(20,0)$ & $185(28,9)$ & $<0,001$ & $72(62,1)$ & $85(47,2)$ & 0,012 \\
\hline Mista & $204(24,2)$ & $177(32,6)$ & 0,001 & $115(59,9)$ & $74(43,3)$ & 0,002 \\
\hline \multicolumn{7}{|l|}{ Idade (anos) } \\
\hline$<19$ & $140(18,8)$ & $135(22,7)$ & 0,077 & $83(65,4)$ & $52(40,6)$ & $<0,001$ \\
\hline $20-29$ & $652(33,2)$ & $834(43,4)$ & $<0,001$ & $350(55,8)$ & $406(49,3)$ & 0,013 \\
\hline $30-39$ & $481(43,6)$ & $800(56,5)$ & $<0,001$ & $297(63,7)$ & $536(67,3)$ & 0,192 \\
\hline 40 ou mais & $46(35,4)$ & $60(51,3)$ & 0,012 & $20(46,5)$ & $41(69,5)$ & 0,019 \\
\hline \multicolumn{7}{|c|}{ Escolaridade materna (anos completos) } \\
\hline $0-4$ & $58(9,7)$ & $41(11,3)$ & 0,420 & $26(50,0)$ & $9(23,1)$ & 0,009 \\
\hline $5-8$ & $293(18,3)$ & $194(18,5)$ & 0,859 & $121(45,7)$ & $55(29,3)$ & $<0,001$ \\
\hline $9-11$ & $621(47,3)$ & $643(46,1)$ & 0,518 & $323(53,9)$ & $240(38,0)$ & $<0,001$ \\
\hline 12 ou mais & $321(81,9)$ & $951(76,4)$ & 0,022 & $258(80,4)$ & $731(77,0)$ & 0,212 \\
\hline \multicolumn{7}{|l|}{ IEN (quintis) } \\
\hline Primeiro (mais pobre) & $58(6,3)$ & $130(16,5)$ & $<0,001$ & $31(63,3)$ & $34(27,2)$ & $<0,001$ \\
\hline Segundo & $150(18,3)$ & $237(29,9)$ & $<0,001$ & $65(47,4)$ & $69(29,9)$ & 0,001 \\
\hline Terceiro & $256(29,9)$ & $348(44,7)$ & $<0,001$ & $97(40,4)$ & $152(44,4)$ & 0,334 \\
\hline Quarto & $362(50,8)$ & $481(61,5)$ & $<0,001$ & $191(54,9)$ & $263(55,1)$ & 0,943 \\
\hline Quinto (mais rico) & $493(78,0)$ & $601(77,6)$ & 0,872 & $366(74,8)$ & $506(84,3)$ & $<0,001$ \\
\hline \multicolumn{7}{|l|}{ Tipo de parto } \\
\hline Normal & $480(22,3)$ & $432(30,2)$ & $<0,001$ & $201(44,9)$ & $130(31,2)$ & $<0,001$ \\
\hline Cesárea & $839(46,9)$ & $1.397(53,4)$ & $<0,001$ & $549(67,4)$ & $905(65,1)$ & 0,281 \\
\hline Total & $1.319(33,4)$ & $1.829(45,1)$ & $<0,001$ & $750(59,4)$ & $1.035(57,3)$ & 0,245 \\
\hline
\end{tabular}

IEN: Indicador Econômico Nacional.

Ainda, com relação à cobertura dos planos de saúde para hospitalização foi possível observar que não houve diferença significativa entre os períodos $(\mathrm{p}=0,245)$. Porém, quando observa-se a cobertura para hospitalização de acordo com cada característica socioeconômica estudada há uma diminuição na cobertura para hospitalização entre aquelas mães de cor preta $(\mathrm{p}=0,012)$ e mista $(\mathrm{p}=0,002)$, diminuição entre mães mais jovens que 30 anos ( $<$ 0,001 e 0,013), entre aquelas que apresentam até 11 anos de estudos ( $\mathrm{p}=0,009 ; \mathrm{p}<0,001 ; \mathrm{p}<0,001)$, entre as do primeiro e segundo quintis de IEN $(\mathrm{p}<$ $0,001)$ e entre as que realizaram o parto normal ( $<<0,001)$ (Tabela 2).

Com relação ao financiamento do parto, o SUS foi responsável por 81,1\% e 68,3\%, já os planos privados de saúde foram responsáveis por $14,4 \%$ e $21,3 \%$ e o pagamento direto por 4,4\% e 10,4\%, respectivamente em 2004 e 2015, equivalendo a uma redução de 12,8p.p. no número de partos financiados pelo SUS ( $<<0,001)$ e um aumento de 6,9p.p. no número de partos financiados por planos privados de saúde ( $\mathrm{p}<0,001)$ e de 6,0p.p. em partos particulares ( $<<0,001)$ (Tabela 3).

Em 2004, entre as mães que não estavam cobertas por um plano de saúde, 96,8\% tiveram seus partos pelo SUS e 50\% das mães que tinham plano de saúde também obtiveram o mesmo financiamento pelo SUS. Em 2015, esses percentuais foram de 90,4\% e 41,6\% (Tabela 3). Entre aquelas com cobertura para hospitalizações, a proporção das que tiveram o parto financiado pelo SUS diminuiu 10,2p.p. (p < 0,001). Entre as mães cujo plano não cobria hospitalizações, essa diminuição foi de 5,5p.p. $(\mathrm{p}=0,021)$ (Tabela 3). 
Tabela 3

Comparação entre as coortes de nascimento de Pelotas, Rio Grande do Sul, Brasil, de 2004 e 2015, quanto à fonte de financiamento do parto, de acordo com variáveis socioeconômicas, cobertura de plano de saúde, cobertura para hospitalização e tipo de parto.

\begin{tabular}{|c|c|c|c|c|c|c|c|c|c|}
\hline \multirow{2}{*}{ Variáveis } & \multicolumn{3}{|c|}{ SUS } & \multicolumn{3}{|c|}{ Plano privado de saúde } & \multicolumn{3}{|c|}{ Particular } \\
\hline & 2004 & 2015 & Valor de p & 2004 & 2015 & Valor de p & 2004 & 2015 & Valor de p \\
\hline \multicolumn{10}{|l|}{ Cor da pele } \\
\hline Branca & $1.832(76,0)$ & $1.745(61,0)$ & $<0,001$ & $436(18,1)$ & $743(25,9)$ & $<0,001$ & $143(5,9)$ & $376(13,1)$ & $<0,001$ \\
\hline Preta & $579(90,5)$ & $553(86,7)$ & 0,033 & $55(8,6)$ & $66(10,3)$ & 0,285 & $6(0,9)$ & $19(3,0)$ & 0,008 \\
\hline Mista & $741(87,9)$ & $468(86,0)$ & 0,309 & $78(9,3)$ & $54(9,9)$ & 0,676 & $24(2,8)$ & $22(4,0)$ & 0,224 \\
\hline \multicolumn{10}{|l|}{ Idade (anos) } \\
\hline$<19$ & $699(93,8)$ & $558(93,8)$ & 0,974 & $36(4,8)$ & $21(3,5)$ & 0,240 & $10(1,3)$ & $16(2,7)$ & 0,076 \\
\hline $20-29$ & $1.615(82,3)$ & $1.409(73,2)$ & $<0,001$ & $266(13,6)$ & $326(16,9)$ & 0,003 & $81(4,1)$ & $189(9,8)$ & $<0,001$ \\
\hline $30-39$ & $783(71,0)$ & $737(52,0)$ & $<0,001$ & $246(22,3)$ & $480(33,9)$ & $<0,001$ & $74(6,7)$ & $200(14,1)$ & $<0,001$ \\
\hline 40 ou mais & $98(76,6)$ & $65(56,0)$ & 0,001 & $21(16,4)$ & $36(31,0)$ & 0,007 & $9(7,0)$ & $15(12,9)$ & 0,122 \\
\hline \multicolumn{10}{|l|}{ Escolaridade materna (anos } \\
\hline \multicolumn{10}{|l|}{ completos) } \\
\hline $0-4$ & $584(97,3)$ & $355(97,8)$ & 0,655 & $10(1,7)$ & $5(1,4)$ & 0,725 & $6(1,0)$ & $3(0,8)$ & 0,786 \\
\hline $5-8$ & $1.520(95,0)$ & $983(94,0)$ & 0,255 & $53(3,3)$ & $28(2,7)$ & 0,353 & $27(1,7)$ & $35(3,3)$ & 0,006 \\
\hline $9-11$ & $983(74,9)$ & $1.088(77,9)$ & 0,070 & $257(19,6)$ & $171(2,2)$ & $<0,001$ & $72(5,5)$ & $138(9,9)$ & $<0,001$ \\
\hline 12 ou mais & $98(25,0)$ & $342(27,4)$ & 0,336 & $229(58,4)$ & $659(52,9)$ & 0,057 & $65(16,6)$ & $244(19,6)$ & 0,183 \\
\hline \multicolumn{10}{|l|}{ IEN (quintis) } \\
\hline Primeiro (mais pobre) & $906(98,5)$ & $756(95,8)$ & 0,001 & $13(1,4)$ & $26(3,3)$ & 0,009 & $1(0,1)$ & $7(0,9)$ & 0,019 \\
\hline Segundo & $782(95,4)$ & $723(91,1)$ & 0,001 & $36(4,4)$ & $47(5,9)$ & 0,164 & $2(0,2)$ & $24(3,0)$ & $<0,001$ \\
\hline Terceiro & $773(90,5)$ & $598(76,8)$ & $<0,001$ & $64(7,5)$ & $101(13,0)$ & $<0,001$ & $17(2,0)$ & $80(10,3)$ & $<0,001$ \\
\hline Quarto & $523(73,5)$ & $410(52,4)$ & $<0,001$ & $145(20,4)$ & $229(29,3)$ & $<0,001$ & $44(6,2)$ & $143(18,3)$ & $<0,001$ \\
\hline Quinto (mais rico) & $212(33,5)$ & $160(20,6)$ & $<0,001$ & $311(49,2)$ & $453(58,4)$ & 0,001 & $109(17,2)$ & $162(20,9)$ & 0,084 \\
\hline \multicolumn{10}{|l|}{ Tipo de parto } \\
\hline Normal & $2.039(94,8)$ & $1.353(94,4)$ & 0,666 & $95(4,4)$ & $62(4,3)$ & 0,900 & $18(0,84)$ & $18(1,3)$ & 0,217 \\
\hline Cesárea & $1.158(64,8)$ & $1.416(54,1)$ & $<0,001$ & $474(26,5)$ & $801(30,6)$ & 0,003 & $156(8,7)$ & $402(15,4)$ & $<0,001$ \\
\hline \multicolumn{10}{|l|}{ Cobertura plano de saúde } \\
\hline Não & $2.537(96,8)$ & $2.006(90,4)$ & $<0,001$ & $24(0,9)$ & $30(1,4)$ & 0,150 & $60(2,3)$ & $184(8,3)$ & $<0,001$ \\
\hline Sim & $659(50,00)$ & $761(41,6)$ & $<0,001$ & $545(41,3)$ & $833(45,5)$ & 0,019 & $114(8,6)$ & $235(12,8)$ & $<0,001$ \\
\hline \multirow{2}{*}{\multicolumn{10}{|c|}{$\begin{array}{l}\text { Cobertura do plano para } \\
\text { hospitalização }\end{array}$}} \\
\hline & & & & & & & & & \\
\hline Não & $418(81,5)$ & $587(76,0)$ & 0,021 & $29(5,6)$ & $29(3,8)$ & 0,109 & $66(12,9)$ & $156(20,2)$ & 0,001 \\
\hline Sim & $187(25,0)$ & $153(14,8)$ & $<0,001$ & $514(68,6)$ & $804(77,7)$ & $<0,001$ & $48(6,4)$ & $78(7,5)$ & 0,359 \\
\hline Total & $3.197(81,1)$ & $2.770(68,3)$ & $<0,001$ & $569(14,4)$ & $863(21,3)$ & $<0,001$ & $174(4,4)$ & $420(10,4)$ & $<0,001$ \\
\hline
\end{tabular}

IEN: Indicador Econômico Nacional.

A Tabela 4 apresenta a média e o desvio padrão (DP) dos valores adicionais com hospitalização e médicos, entre mães cobertas por plano de saúde, e valores pagos para hospitalização e médicos entre aquelas mulheres que optaram por parto com financiamento particular.

Dentre as mães cuja fonte de financiamento para o parto era o plano privado de saúde, houve um aumento significativo nos gastos adicionais com hospitalização entre os dois estudos, de R\$ 60,38 $(288,66)$ em 2004 para R\$171,15 (957,07) em 2015 ( $\mathrm{p}=0,008)$. O mesmo foi observado para os gastos adicionais com médicos, que aumentaram de $\mathrm{R} \$ 191,60(612,86)$ em 2004 para $\mathrm{R} \$ 1.424,80(4.459,16)$ em 2015 ( $\mathrm{p}<0,001)$ (Tabela 4).

Entre as mães cuja fonte de financiamento para o parto foi considerado o desembolso particular, não foi observada diferença significativa entre os estudos para gastos com hospitalização ou com médicos para as características pesquisadas. Os gastos com médicos só apresentaram diferença significativa no quintil mais rico do IEN $(\mathrm{p}=0,005)$ (Tabela 4$)$. 
Tabela 4

Média e desvio padrão (DP) de gastos com hospitalização e médicos entre mulheres cujas fontes de financiamento para o parto foram plano privado de saúde ou particular, de acordo com variáveis socioeconômicas. Pelotas, Rio Grande do Sul, Brasil.

\begin{tabular}{|c|c|c|c|c|c|c|}
\hline & \multicolumn{3}{|c|}{ Plano privado de saúde } & \multicolumn{3}{|c|}{ Particulares } \\
\hline & 2004 & 2015 & Valor de $p$ * & 2004 & 2015 & Valor de $p$ * \\
\hline & $R \$(D P)$ & $R \$(D P)$ & & $\mathrm{R} \$(D P)$ & $R \$(D P)$ & \\
\hline \multicolumn{7}{|c|}{ Gastos com hospitalização } \\
\hline \multicolumn{7}{|l|}{ Cor da pele } \\
\hline Branca & $72,70(309,9)$ & $194,11(1028,0)$ & 0,017 & $1.294,00(911,19)$ & $1.735,79(5.339,27)$ & 0,334 \\
\hline Preta & $47,25(309,11)$ & $20,48(117,41)$ & 0,517 & $839,13(334,03)$ & $1.298,86(1.537,40)$ & 0,519 \\
\hline Mista & $0,50(4,44)$ & $39,45(180,09)$ & 0,060 & $1.575,95(861,74)$ & $1.372,42(1.523,00)$ & 0,582 \\
\hline \multicolumn{7}{|l|}{ Idade (anos) } \\
\hline$<19$ & $57,23(343,38)$ & $0,00(0,00)$ ** & 0,450 & $1.332,42(753,42)$ & $1.216,61(1.490,14)$ & 0,822 \\
\hline $20-29$ & $37,36(214,88)$ & $130,50(573,09)$ & 0,013 & $1.251,47(848,39)$ & $2.005,31(7.353,29)$ & 0,371 \\
\hline $30-39$ & $82,47(344,03)$ & $211,61(1.185,79)$ & 0,096 & $1.359,83(862,67)$ & $1.428,62(1.628,91)$ & 0,735 \\
\hline 40 ou mais & $98,51(289,19)$ & $99,72(453,47)$ & 0,991 & $1.518,06(1.610,32)$ & $1.619,34(1.490,37)$ & 0,877 \\
\hline \multicolumn{7}{|c|}{$\begin{array}{l}\text { Escolaridade materna (anos } \\
\text { completos) }\end{array}$} \\
\hline $0-4$ & $0,00(0,00)$ & $0,00(0,00)$ & ** & $1.723,67(1.118,39)$ & $1.065,93(1.846,24)$ & 0,518 \\
\hline $5-8$ & $116(451,75)$ & $123,91(596,46)$ & 0,953 & $1.287,20(827,91)$ & $1.431,33(1.739,89)$ & 0,698 \\
\hline $9-11$ & $36,84(210,59)$ & $133,76(575,42)$ & 0,014 & $1.325,87(970,15)$ & $1.533,02(1.580,47)$ & 0,322 \\
\hline 12 ou mais & $80,72(330,43)$ & $184,16(1.048,32)$ & 0,143 & $1.272,88(799,52)$ & $1.818,16(6.520,31)$ & 0,509 \\
\hline \multirow{2}{*}{\multicolumn{7}{|c|}{$\begin{array}{l}\text { Renda familiar (salários } \\
\text { mínimos) }\end{array}$}} \\
\hline & & & & & & \\
\hline$<1$ & $51,28(303,84)$ & $52,92(167,35)$ & 0,987 & $1.162,16(292,94)$ & $2.697,71(2.738,17)$ & 0,038 \\
\hline $1-2,9$ & $60,64(339,83)$ & $136,91(578,04)$ & 0,119 & $1.328,69(916,26)$ & $1.557,77(1.444,30)$ & 0,342 \\
\hline $3-5,9$ & $71,17(302,45)$ & $164,45(740,19)$ & 0,119 & $1.436,64(1.116,05)$ & $1.443,46(1.595,03)$ & 0,977 \\
\hline $6-9,9$ & $52,24(166,52)$ & $236,50(1.665,12)$ & 0,294 & $1.289,93(863,71)$ & $2.940,66(1.2085,58)$ & 0,443 \\
\hline 10 ou mais & $49,38(134,70)$ & $154,40(437,31)$ & 0,109 & $1.183,73(671,08)$ & $909,41(987,66)$ & 0,240 \\
\hline \multicolumn{7}{|l|}{ IEN } \\
\hline Primeiro (mais pobre) & $0,00(0,00)$ & $126,46(614,24)$ & 0,487 & 920,07 ** & $2.457,61(1.486,48)$ & ** \\
\hline Segundo & $84,19(396,18)$ & $85,58(487,67)$ & 0,989 & $1946,42(1.213,57)$ & $1.513,05(1.589,99)$ & 0,712 \\
\hline Terceiro & $76,40(297,92)$ & $113,70(515,22)$ & 0,602 & $1214,38(687,41)$ & $1.303,75(1.701,29)$ & 0,832 \\
\hline Quarto & $40,79(258,08)$ & $248,52(1.555,59)$ & 0,113 & $1429,33(948,19)$ & $1.594,87(1.752,44)$ & 0,562 \\
\hline Quinto (mais rico) & $66,02(292,35)$ & $159,28(645,88)$ & 0,017 & $1283,11(912,90)$ & $1.958,37(7.889,79)$ & 0,384 \\
\hline \multicolumn{7}{|l|}{ Tipo de parto } \\
\hline Normal & $10,67(61,22)$ & $37,40(114,76)$ & 0,059 & $1.146,87(1.028,21)$ & $1.417,10(1.522,65)$ & 0,537 \\
\hline Cesárea & $70,43(314,40)$ & $181,51(992,21)$ & 0,018 & $1.337,27(881,19)$ & $1.698,94(5.178,38)$ & 0,397 \\
\hline Total & $60,38(288,66)$ & $171,15(957,07)$ & 0,008 & $1.316,75(896,70)$ & $1.686,86(5.075,53)$ & 0,349 \\
\hline \multicolumn{7}{|l|}{ Gastos com médicos } \\
\hline \multicolumn{7}{|l|}{ Cor da pele } \\
\hline Branca & $230,05(685,76)$ & $1556(4.766,46)$ & $<0,001$ & $2.651,81(1.762,04)$ & $2.825,72(2.185,77)$ & 0,3947 \\
\hline Preta & $71,78(224,89)$ & $506,77(1.079,92)$ & 0,004 & $2.356,78(1.253,32)$ & $2.286,27(1.718,77)$ & 0,927 \\
\hline Mista & $61,48(208,78)$ & $730(1.454,26)$ & $<0,001$ & $1.760,10(1.405,33)$ & $3.248,31(2.578,16)$ & 0,020 \\
\hline \multicolumn{7}{|l|}{ Idade (anos) } \\
\hline$<19$ & $128,56(358,70)$ & $147,31(585,96)$ & 0,881 & $3.070,41(1.609,91)$ & $3.402,88(2.669,09)$ & 0,726 \\
\hline $20-29$ & $169,72(531,79)$ & $982,98(2.182,90)$ & $<0,001$ & $2.328,90(1.566,95)$ & $2.664,69(2.119,98)$ & 0,203 \\
\hline $30-39$ & $216,31(696,26)$ & $1.793,00(5.656,23)$ & $<0,001$ & $2.618,40(1.926,46)$ & $2.961,05(2.196,14)$ & 0,237 \\
\hline 40 ou mais & $288,77(855,91)$ & $1.261,51(1.656,09)$ & 0,015 & $2.834,00(1.242,03)$ & $2.699,79(615,49)$ & 0,877 \\
\hline
\end{tabular}

(continua) 
Tabela 4 (continuação)

\begin{tabular}{|c|c|c|c|c|c|c|}
\hline & \multicolumn{3}{|c|}{ Plano privado de saúde } & \multicolumn{3}{|c|}{ Particulares } \\
\hline & 2004 & 2015 & Valor de $p$ * & 2004 & 2015 & Valor de $p$ * \\
\hline & $R \$(D P)$ & $R \$(D P)$ & & $R \$(D P)$ & $R \$(D P)$ & \\
\hline \multicolumn{7}{|l|}{$\begin{array}{l}\text { Escolaridade materna (anos } \\
\text { completos) }\end{array}$} \\
\hline $0-4$ & $108.52(281,47)$ & $0,00(0,00)$ & 0,413 & $2.146,20(1.268,48)$ & $2.676,55(2.537,79)$ & 0,699 \\
\hline $5-8$ & $145,10(404,52)$ & $56,01(218,78)$ & 0,283 & $2.392,45(1.388,06)$ & $2.139,81(2.190,58)$ & 0,603 \\
\hline $9-11$ & $133,15(420,65)$ & $784,94(4.079,09)$ & 0,015 & $2.368,42(1.283,31)$ & $2.539,66(2.108,19)$ & 0,529 \\
\hline 12 ou mais & $282,51(821,80)$ & $1.659,80(4.635,85)$ & $<0,001$ & $2.801,53(2.224,34)$ & $3.104,03(2.197,64)$ & 0,326 \\
\hline \multirow{2}{*}{\multicolumn{7}{|c|}{$\begin{array}{l}\text { Renda familiar (salários } \\
\text { mínimos) }\end{array}$}} \\
\hline & & & & & & \\
\hline$<1$ & $282,06(665,19)$ & $1.052,80(1.386,14)$ & 0,009 & $2.683,97(636,37)$ & $2.305,81(3.114,05)$ & 0,648 \\
\hline $1-2,9$ & $131,19(430,45)$ & $1.080,49(4.432,41)$ & 0,002 & $2.083,94(1.307,73)$ & $2.382,22(2.21,39)$ & 0,393 \\
\hline $3-5,9$ & $165,89(623,43)$ & $832,71(1.329,14)$ & $<0,001$ & $2.279,07(1.432,85)$ & $2.581,61(148,12)$ & 0,320 \\
\hline $6-9,9$ & $196,48(543,70)$ & $1.947,49(7.815,57)$ & 0,034 & $2.539,88(1.597,95)$ & $3.097,52(2.328,06)$ & 0,212 \\
\hline 10 ou mais & $460,37(115,60)$ & $2.014,06(2.034,69)$ & $<0,001$ & $3.529,59(2.382,62)$ & $4.230,66(2.115,09)$ & 0,252 \\
\hline \multicolumn{7}{|l|}{ IEN } \\
\hline Primeiro (mais pobre) & $59,21(164,07)$ & $254,82(665,35)$ & 0,306 & $1.955,15 * *$ & $2.063,99(2.706,77)$ & ** \\
\hline Segundo & $53,32(180,25)$ & $194,89(643,46)$ & 0,204 & $1.474,14(2.084,75)$ & $1.581,20(1.803,00)$ & 0,937 \\
\hline Terceiro & $138,53(291,86)$ & $643,15(1.281,21)$ & 0,002 & $2.507,18(1.395,29)$ & $2.761,71(1.888,97)$ & 0,611 \\
\hline Quarto & $92,59(294,83)$ & $1.289,24(4.135,22)$ & $<0,001$ & $2.375,80(1.333,39)$ & $2.502,28(2.078,43)$ & 0,704 \\
\hline Quinto (mais rico) & $270,50(782,27)$ & $1.864,88(5.314,68)$ & $<0,001$ & $2.605,58(1.909,65)$ & $3.356,86(2.311,85)$ & 0,005 \\
\hline \multicolumn{7}{|l|}{ Tipo de parto } \\
\hline Normal & $460,37(1115,60)$ & $2.014,06(2.034,69)$ & $<0,001$ & $1.720,83(890,12)$ & $2.689,06(2.075,55)$ & 0,077 \\
\hline Cesárea & $93,82(497,46)$ & $1.183,44(1.686,87)$ & $<0,001$ & $2.614,89(1.768,69)$ & $2.841,73(2.194,74)$ & 0,250 \\
\hline Total & $191,60(612,86)$ & $1.424,80(4.459,16)$ & $<0,001$ & $2.521,87(1.718,76)$ & $2.835,19(2.187,63)$ & 0,093 \\
\hline
\end{tabular}

IEN: Indicador Econômico Nacional.

* Valor de p refere-se ao teste t de Student para comparação de médias;

** Não tem desvio padrão e não é possível calcular a diferença entre as médias. 


\section{Discussão}

Este trabalho comparou os desembolsos relacionados ao parto entre as coortes de nascimento de Pelotas de 2004 e 2015. O SUS foi o financiador da maioria dos partos das duas coortes, embora tenha havido uma diminuição no período, com consequente aumento do número de partos cobertos por planos de saúde e particulares. Entre 2004 e 2015, houve aumento da renda familiar e aumento na posse de planos de saúde, em todos os grupos econômicos. Além disso, a cobertura para hospitalizações dos planos das famílias do primeiro e segundo quintis mais pobres diminuiu, já a das demais famílias aumentou. Dentre as mães cobertas por planos de saúde, as despesas com atendimento médico aumentaram em cerca de sete vezes no período.

O aumento nos valores gastos com o parto pode ser um sinal de alerta, pois em alguns países, como a Índia, os gastos com a assistência ao parto podem levar a valores catastróficos 17,18. No Canadá, mesmo em hospitais públicos, os valores com serviços de parto podem ser altos para a população mais pobre. Neste estudo, as despesas durante o parto foram significativamente associadas à educação e ao tipo de parto das entrevistadas 19.

Outro ponto importante é o aumento na cobertura por plano de saúde. O aumento na proporção de mães cobertas por planos de saúde pode estar relacionado ao aumento do poder aquisitivo da população no mesmo período. A PNS de 2013 mostrou que a posse de planos de saúde no Brasil é de cerca de 33\% na Região Sul 6, inferior ao valor encontrado neste trabalho. Desde a PNAD de 1998, estudos que avaliam a cobertura por plano de saúde mostram uma tendência de aumento na posse de planos 20 . A melhora do nível socioeconômico das mulheres no período estudado pode ter contribuído para o aumento da posse de planos de saúde 21.

Com relação ao financiamento do parto, houve diminuição do número de partos financiados pelo SUS e aumento de partos realizados por meio de plano privado de saúde e por pagamento direto privado. Estudos realizados em Ribeirão Preto (São Paulo) 21, Maringá (Paraná) 22 e São Leopoldo (Rio Grande do Sul) 23 mostraram resultados semelhantes. Nesse período também houve aumento da prevalência de cesáreas entre as mulheres com parto por financiamento privado e por planos de saúde pré-pagamento e diminuição entre as atendidas no SUS, o que é consistente com os achados de outros trabalhos 21,24 .

Uma possível explicação para o aumento em cerca de sete vezes nos gastos com médicos entre os dois anos pode ser a normativa sobre a possibilidade dos médicos cobrarem a taxa de disponibilidade para o acompanhamento do parto ${ }^{8}$. A cobrança dessa taxa por médicos conveniados é considerada ilegal no Brasil, porém muitos deles acabaram optando por se desvincular do plano de saúde na parte obstétrica para poder efetuar a cobrança, deixando a cargo da mãe a escolha por ser acompanhada pelo obstetra de sua preferência 8,11. Outros estudos econômicos são necessários para melhor entender esses achados, pois esses gastos podem impactar na escolha do tipo de parto, na escolha do obstetra de acordo com as possibilidades maternas.

Este trabalho tem pontos positivos e algumas limitações. A inclusão de todos os partos de mães que vivem na área urbana de Pelotas e a baixa taxa de perdas nas duas coortes são pontos positivos do estudo. $\mathrm{O}$ tamanho da amostra é grande, permitindo estratificações necessárias para as análises. Além disso, o período de recordatório para informações relacionadas aos desembolsos maternos com parto e plano de saúde foi curto (três meses) e igual para as mães das duas coortes, contribuindo para a qualidade e comparabilidade dos dados. Deve-se notar, no entanto, que as informações sobre desembolsos e características dos planos de saúde foram obtidas na entrevista com as mães, que talvez não estejam cientes dos detalhes exatos da cobertura e dos custos. Como na maioria das pesquisas, as informações sobre os desembolsos foram baseadas em recordatório, estando assim sujeitas a erros. Além disso, algumas mulheres podem ter cobertura apenas ambulatorial de seus planos, podendo superestimar os dados referentes aos partos particulares.

Este estudo indica que apesar da diminuição dos partos realizados pelo SUS no período, a maioria dos nascimentos ainda é financiado pelo sistema público de saúde brasileiro, que continua desempenhando um papel importante na redução do desembolso e na promoção de equidade em saúde. Houve um aumento significativo do desembolso materno entre aquelas que possuíam plano de saúde, principalmente no estrato mais rico. 


\section{Colaboradores}

M. C. Guttier e C. A. O. Tejada contribuíram na concepção e planejamento, redação, análise e interpretação dos dados, revisão crítica do conteúdo e aprovação da versão final. F. C. Wehrmeister, M. F. Silveira, M. R. Domingues, A. J. D. Barros, I. S. Santos, A. Matijasevich, D. G. Bassani e A. D. Bertoldi contribuíram na concepção e planejamento, revisão crítica do conteúdo e aprovação da versão final.

\section{Informações adicionais}

ORCID: Marília Cruz Guttier (0000-0002-28706334); Cesar Augusto Oviedo Tejada (0000-00028120-5563); Fernando C. Wehrmeister (00000001-7137-1747); Mariângela Freitas Silveira (0000-0002-2861-7139); Marlos R. Domingues (0000-0002-2503-2944); Aluísio J. D. Barros (00000002-2022-8729); Iná S. Santos (0000-0003-12589249); Alícia Matijasevich (0000-0003-0060-1589); Diego G. Bassani (0000-0001-6704-3820); Andréa Dâmaso Bertoldi (0000-0002-4680-3197).

\section{Agradecimentos}

Este artigo foi realizado com dados do estudo Coorte de Nascimentos de Pelotas, 2004, conduzido pelo Programa de Pós-graduação em Epidemiologia da Universidade Federal de Pelotas, com o apoio da Associação Brasileira de Saúde Coletiva (ABRASCO). De 2009 a 2013, a coorte de nascimentos de 2004 foi financiada pelo Wellcome Trust. Fases anteriores do estudo foram financiadas pela Organização Mundial da Saúde, Programa de Apoio a Núcleos de Excelência (PRONEX), Conselho Nacional de Desenvolvimento Científico e Tecnológico (CNPq), Ministério da Saúde e Pastoral da Criança. Este trabalho foi realizado com dados do estudo Coorte de Nascimentos de Pelotas, 2015, conduzido pelo Programa de Pós-graduação em Epidemiologia da Universidade Federal de Pelotas, com o apoio da ABRASCO. A Coorte de Pelotas de 2015 foi financiada pela Wellcome Trust (095582). Foram recebidos também financiamentos para os seguimentos específicos do $\mathrm{CNPq}$ e da Fundação de Amparo à Pesquisa do Estado do Rio Grande do Sul (FAPERGS).

\section{Referências}

1. Dieleman J, Campbell M, Chapin A, Eldrenkamp E, Fan VY, Haakenstad A, et al. Evolution and patterns of global health financing 1995-2014: development assistance for health, and government, prepaid private, and out-ofpocket health spending in 184 countries. Lancet 2017; 389:1981-2004.

2. Barros AJ, Bertoldi AD. Out-of-pocket health expenditure in a population covered by the Family Health Program in Brazil. Int J Epidemiol 2008; 37:758-65.

3. Instituto Brasileiro de Geografia e Estatística. Pesquisa de orçamentos familiares 2017-2018: primeiros resultados. Rio de Janeiro: Instituto Brasileiro de Geografia e Estatística; 2019.

4. Albuquerque VMT, Tavares CA. Avaliação de Indicadores de Medicamentos: importância para a qualidade na prescrição médica. Revista Brasileira de Farmácia Hospitalar e Serviços de Saúde 2011; 2:31-5.

5. Almeida G, Sarti FM, Ferreira FF, Diaz MDM, Campino ACC. Analysis of the evolution and determinants of income-related inequalities in the Brazilian health system, 1998-2008. Rev Panam Salud Pública 2013; 33:90-7.

6. Malta DC, Stopa SR, Pereira CA, Szwarcwald CL, Oliveira M, Reis AC. Cobertura de Planos de saúde na população brasileira, segundo a Pesquisa Nacional de Saúde, 2013. Ciênc Saúde Colet 2017; 22:179-90.

7. Agência Nacional de Saúde Suplementar. Caderno de Informação da Saúde Suplementar: Beneficiários, Operadoras e Planos 2018; 12:1-57.

8. Agência Nacional de Saúde. Resolução Normativa no 368, de 6 de janeiro de 2015. Dispõe sobre o direito de acesso à informação das beneficiárias aos percentuais de cirurgias cesáreas e de partos normais, por operadora, por estabelecimento de saúde e por médico e sobre a utilização do partograma, do cartão da gestante e da carta de informação à gestante no âmbito da saúde suplementar. Diário Oficial da União 2015; 6 jul.

9. Ministério da Saúde. Portaria no 2.418 , de 2 de dezembro de 2005. Regulamenta, em conformidade com o art. 1o da Lei no 11.108 , de 7 de abril de 2005, a presença de acompanhante para mulheres em trabalho de parto, parto e pós-parto imediato nos hospitais públicos e conveniados com o Sistema Único de Saúde SUS. Diário Oficial da União 2005; 6 dez.

10. Ministério da Saúde. Humanização do parto e do nascimento. Brasília: Ministério da Saúde; 2014. (Cadernos HumanizaSUS, 4).

11. Barbosa JMDC, Baggio AC. A cobrança da taxa de disponibilidade pelos médicos obstetras. Percurso 2015; 15:28-51.

12. Agência Nacional de Saúde Suplementar. Grupo técnico para debate sobre parecer CFM no 39/12. Brasília: Agência Nacional de Saúde Suplementar; 2013. (Ofício no 59/2013/PRESI). 
13. Hallal PC, Bertoldi AD, Domingues MR, Silveira MF, Demarco FF, Silva ICM, et al. Cohort profile: the 2015 Pelotas (Brazil) Birth Cohort Study. Int J Epidemiol 2018; 47:1048-h.

14. Santos IS, Barros AJ, Matijasevich A, Zanini R, Chrestani Cesar MA, Camargo-Figuera FA, et al. Cohort profile update: 2004 Pelotas (Brazil) Birth Cohort Study. Body composition, mental health and genetic assessment at the 6 years follow-up. Int J Epidemiol 2014; 43:1437-a-f.

15. Santos IS, Barros AJ, Matijasevich A, Domingues MR, Barros FC, Victora CG. Cohort profile: the 2004 Pelotas (Brazil) birth cohort study. Int J Epidemiol 2011; 40:1461-8.

16. Barros AJ, Victora CG. A nationwide wealth score based on the 2000 Brazilian demographic census. Rev Saúde Pública 2005; 39:523-9.

17. Goli S, Rammohan A. Out-of-pocket expenditure on maternity care for hospital births in Uttar Pradesh, India. Health Econ Rev 2018; 8:5.

18. Govil D, Purohit N, Gupta SD, Mohanty SK. Out-of-pocket expenditure on prenatal and natal care post Janani Suraksha Yojana: a case from Rajasthan, India. J Health Popul Nutr 2016; 35:15.

19. Tellis SB, Rent PD, Dmello MK. Utilization of antenatal care and out of pocket expenditure on delivery care in Dakshina Kannada. Int J Community Med Public Health 2018; 5:3553-8.
20. Viacava F, Bellido JG. Condições de saúde, acesso a serviços e fontes de pagamento, segundo inquéritos domiciliares. Ciênc Saúde Colet 2016; 21:351-70.

21. Yazlle MEHD, Rocha JSY, Mendes MC, Patta MC, Marcolin AC, Azevedo GD. Incidência de cesáreas segundo fonte de financiamento da assistência ao parto. Rev Saúde Pública 2001; 35:202-6.

22. Paris GF, Monteschio LVC, Oliveira RR, Latorre MRDO, Pelloso SM, Mathias TAF. Tendência temporal da via de parto de acordo com a fonte de financiamento. Rev Bras Ginecol Obstet 2014; 36:548-54,

23. Schreiner M, Costa JSD, Olinto MTA, Meneghel SN. Assistência ao parto em São Leopoldo - RS: um estudo de base populacional. Ciênc Saúde Colet 2010; 15 Suppl 1:1411-6.

24. Barros AJD, Santos IS, Matijasevich A, Domingues MR, Silveira M, Barros FC, et al. Patterns of deliveries in a Brazilian birth cohort: almost universal cesarean sections for the better-off. Rev Saúde Pública 2011; 45:635-43. 


\section{Abstract}

Although most childbirth care in Brazil is financed by the Brazilian Unified National Health System (SUS), there are out-of-pocket expenditures (private personal costs) involved in births. This study aims to compare maternal out-of-pocket expenditures in births of children from the Pelotas Birth Cohorts of 2004 and 2015. The study drew on information collected right after birth and at three months of age. The target variables include sociodemographic and economic data, private health plan coverage, and expenditures related to the birth. Values from 2004 were adjusted to 2015 by the general price index. There was an increase in private health plan coverage from $33.4 \%$ (95\% CI: 31.9-34.9) to 45.1\% (95\%IC: 43.6-46.7) in the target period, directly associated with the families' socioeconomic status $(p<0.001)$. There was an increase in mean expenditures on hospitalization for the birth, from BRL $60.38(S D=288.66)$ to $B R L$ $171.15(S D=957.07)$, and in additional medical expenditures, from BRL $191.60(S D=612.86)$ to $B R L 1,424.80(S D=4,459.16)$ among mothers admitted to hospital under their private health plans (and there was no significant difference in these expenditures for mothers that opted for direct payment). There was an important increase in expenditures for childbirth care, especially among mothers admitted to hospital under private health plans.

Health Equity; Obstetric Delivery; Maternal Health Services; Health Expenditures;

Longitudinal Studies

\section{Resumen}

A pesar de que la mayoría de los partos en Brasil esté financiado por el Sistema Único de Salud, existen gastos directos (personales privados) implicados en el nacimiento. Este estudio tiene como objetivo comparar el desembolso materno para financiar los partos de los niños, pertenecientes a las cohortes de nacimientos de Pelotas desde el 2004 al 2015. Se utilizó información recogida tras el nacimiento y a los tres meses de edad. Las variables analizadas incluyen información sociodemográfica, económica, cobertura con plan privado de salud y gastos relacionados con el parto. Los valores de 2004 se ajustaron por el Índice Nacional de Precios al Consumidor Amplio. Se observó un aumento en la posesión de planes de salud de un 33,4\% (IC95\%: 31,9-34,9) a un 45, 1\% (IC95\%: 43,6-46,7) durante el período analizado y este se mostró directamente asociado a la posición económica de las familias $(p<0,001)$. Se produjo un aumento en la media de los gastos con hospitalización para el parto de BRL 60,38 $(D E=288,66)$ a $B R L 171,15(D E=957,07), y$ en los gastos adicionales con médicos, de BRL 191,60 (DE =612,86) a BRL 1.424,80 $(D E=4.459,16)$ entre las madres que estaban internadas por el plan privado de salud (y no hubo diferencia significativa en estos gastos entre las madres que optaron por el parto particular). Hubo un aumento importante en el gasto con asistencia al parto, principalmente, entre madres que estuvieron internadas para el parto mediante un plan privado de salud.

Equidad em Salud; Parto Obstétrico; Servicios de Salud Materna; Gastos en Salud; Estudios Longitudinales
Recebido em 25/Jun/2019

Versão final reapresentada em 30/Out/2019 Aprovado em 02/Dez/2019 